10 years ESJ

Special edition

\title{
Estrategias de Mercadotecnia Gastronómica para Restaurantes de Puebla en Tiempos de Pandemia por COVID-19. Estudio de Caso: Generación Z
}

\author{
Teresa Galdys Cerón Carrillo \\ Yesbek Rocío Morales Paredes \\ Norma Angélica Santiesteban López
}

Benemérita Universidad Autónoma de Puebla, Mexico

Doi: $10.19044 /$ esj.2021.v17n4p242

Submitted: 28 October 2020

Accepted: 10 December 2020

Published: 07 February 2021
Copyright 2021 Author(s)

Under Creative Commons BY-NC-ND

4.0 OPEN ACCESS

Cite As:

Cerón Carrillo G.T., Morales Paredes Y.R. \& Santiesteban López N.A. (2021). Estrategias de Mercadotecnia Gastronómica para Restaurantes de Puebla en Tiempos de Pandemia por COVID-19. Estudio de Caso: Generación Z. European Scientific Journal, ESJ, 17(4), 242. https://doi.org/10.19044/esj.2021.v17n4p242

\section{Resumen}

La pandemia por COVID-19 ha propiciado que los restaurantes en la Ciudad de Puebla reduzcan el flujo de clientes, por lo que implementar las nuevas disposiciones de higiene y sanidad marcadas en el Protocolo para la implementación de medidas preventivas ante COVID-19 en restaurantes de la Secretaría de protección civil y gestión integral de riesgos de la Ciudad de Puebla y el Protocolo de prevención COVID-19 "Mesa segura" de la CANIRAC, resulta imprescindible para la reapertura en la nueva normalidad, así, el uso de estrategias de mercadotecnia gastronómica se consolida como el medio adecuado para atraer a clientes de la Generación $\mathrm{Z}$ que buscan experiencias seguras, novedosas, saludables y de bajo impacto ambiental, que les brinden valor por lo que pagan, utilizando las herramientas virtuales que se adapten a sus necesidades y gustos. Para lo anterior se realizó un estudio transversal, no experimental y un cuestionario en línea de 20 ítems el cual se distribuyó vía redes sociales entre 616 participantes pertenecientes a la Generación Z, logrando así el objetivo de generar estrategias de mercadotecnia gastronómica para un consumo seguro en restaurantes de Puebla frente a la nueva normalidad. Los resultados obtenidos revelaron que el segmento mencionado está dispuesto a consumir en restaurantes siempre y cuando se 
cuenten con las medidas de seguridad e higiene propuestas por las autoridades correspondientes, así como, cumplan con sus expectativas y exigencias.

Palabras clave: Mercadotecnia gastronómica, generación Z, restaurantes, Puebla

\title{
Gastronomic Marketing Strategies for Restaurants in Puebla during COVID-19 Pandemic: A Case Study of Generation Z
}

\author{
Teresa Galdys Cerón Carrillo \\ Yesbek Rocío Morales Paredes \\ Norma Angélica Santiesteban López \\ Benemérita Universidad Autónoma de Puebla, Mexico
}

\begin{abstract}
The COVID-19 pandemic has led restaurants in Puebla City to reduce the flow of customers. This is why it is essential to implement the new hygiene and health provisions following the Protocol for the preventive measures against COVID-19 in restaurants as proposed by the Ministry of Civil Protection and Integral Risk Management of Puebla as well as CANIRAC's COVID-19 "Safe Table" Prevention Protocol. The use of gastronomic marketing strategies is consolidated as the appropriate means to attract Generation $\mathrm{Z}$ clients who are looking for safer, innovative, healthy and lowenvironmental impact experiences that provides value for what they pay, while using virtual tools that adapt to their needs and tastes. Based on the aforementioned, a cross-sectional and non-experimental study was carried out, and an online questionnaire of 20 items was distributed via social networks among 616 participants belonging to Generation Z. This paper focuses on generating gastronomic marketing strategies for safer consumption in Puebla restaurants that have adopted the new normal. Results obtained revealed that this segment are willing to eat in restaurants as long as they have safety and hygiene measures proposed by the corresponding authorities, in addition to meeting their expectations and demands.
\end{abstract}

Keywords: Gastronomic marketing, Z generation, restaurants, Puebla

\section{Introduction}

La generación Z "presta mucha atención a las opiniones de las personas que ya han experimentado por cuenta propia el alimento o servicio que desean consumir, indagando primero las opiniones de los clientes de dicha empresa en redes sociales o portales web" (Carvalho dos Santos Claro et al., 2015). 
La generación $\mathrm{Z}$ que visita los restaurantes muestra una tendencia de consumo que no se enfoca en la posesión de los bienes y el lujo; sino en el acceso a estos para lograr su objetivo de vivir nuevas experiencias y de acumular momentos y emociones únicas que marquen su manera de pensar y sentir.

Dicha conducta se ve reflejada en su forma de consumir constituyendo esta, la razón por la que las nuevas tipologías de acudir a este tipo de establecimientos está ganando una fuerte aceptación entre los jóvenes de esta generación.

Debido a que este tipo de servicio de alimentos les ofrece dos factores muy importantes que los mismos buscan cuando eligen un lugar donde ir a consumir alimentos, una es vivir experiencias únicas y diferentes que les permitan involucrarse más con la comunidad y al mismo tiempo ahorrar dinero y sacarle el máximo provecho al mismo (Bell, 2015).

Los jóvenes de la generación $\mathrm{Z}$ o centennials son muy críticos, exigentes y con mucha información a la mano por lo que desean acudir al establecimiento que les ofrezca más por menos. Como han crecido en una época de crisis económica han aprendido a ser muy cautelosos con su dinero y se aseguran de gastarlo tan solo en aquello que les ayude a alcanzar sus objetivos de bienestar obteniendo siempre la mayor conveniencia y comodidad al menor costo en todas sus transacciones (Barton et al., 2012).

Con los resultados obtenido de la investigación se pudo deducir que los centennials muestran una preferencia en el servicio de alimentación más inclinada hacia la gastronomía local que les permita acercarse a través de la misma a la cultura de la ciudad, probando nuevos ingredientes y preparaciones, prefieren un estilo de comida más sano y más local, y que muestran una preferencia a consumir en aquellos establecimientos que les ofrezcan alimentos orgánicos, sin conservantes ni aditivos, que apoyen la producción local, utilicen los ingredientes de la zona y conserven y potencien la gastronomía típica (Bell, 2015).

Esta es la razón, por la que hoy en día los restaurantes requieren crear nuevas estrategias de marketing para llegar, atraer y conquistar a los consumidores de esta generación

Actualmente ya conforman un segmento de mercado muy atractivo para las empresas de este sector y tienen un fuerte nivel de influencia en los consumidores en general. Por sí solos tienen la capacidad de generar marketing de influencia, provocar cambios en los mercados y marcar tendencias de compra, haciéndolo de manera especial en la industria de los restaurantes, por ser una de sus actividades preferidas (Ponce, 2017).

Las redes sociales hoy en día "son las herramientas con mayor crecimiento y popularidad desde la aparición del marketing digital" (España et al., 2020). Razón por la que se han convertido en plataformas indispensables 
y puntos estratégicos de conexión para las empresas con sus consumidores, especialmente con los de la generación Z (Campos, 2019), quienes como se ha visto y confirmado a lo largo de esta investigación hacen uso intensivo de las mismas, las consideran parte integral de sus vidas, y recurren a ellas para consultar y elegir los destinos y establecimientos que preparan alimentos (Campos, 2019).

El marketing gastronómico de esta generación es particularmente atraído por los establecimientos que son responsables social y ambientalmente, que les ofrecen comida saludable y típica del lugar, y sobretodo que les invitan a cooperar e involucrarse con estos factores.

Debido a lo anteriormente mencionado el objetivo de la presente investigación es generar estrategias de mercadotecnia gastronómica para un consumo seguro en restaurantes de Puebla frente a la nueva normalidad, que atraigan a consumidores del segmento de mercado Generación Z.

\section{La industria restaurantera frente al COVID-19}

Durante la pandemia por COVID-19 una de las industrias más afectadas ha sido la restaurantera, misma que hasta el 2019 generaba alrededor de 700 mil empleos, colocándola como la mayor empleadora de México, representando cerca del 1.7 del Producto Interno Bruto (PIB) nacional y el $13 \%$ del PIB turístico (Aranda, 2019). Hasta el año pasado, según el Directorio Estadístico Nacional de Unidades Económicas (DENUE) del Instituto Nacional de Estadística y Geografía (INEGI, 2019), eran 555,354 unidades económicas las que laboraban en la República Mexicana, hoy 54,154 unidades han cerrado sus puertas.

De acuerdo a Germán González, vicepresidente de a Cámara Nacional de la Industria Restaurantera y Alimentos Condimentados (CANIRAC) entre el 20 y $25 \%$ de los restaurantes en México cerrarían sus puertas de forma definitiva (Lozano, 2020), pues no podrían soportar el largo periodo de confinamiento sin ganancias por las bajas o nulas ventas; provocando pérdidas de más de 75 mil millones de pesos a nivel nacional (Raziel, 2020). Por su parte, Francisco Fernández Alonso, presidente de la CANIRAC indicó que Puebla es uno de los estados más afectados en toda la República Mexicana.

De acuerdo al DENUE (INEGI, 2019) el estado de Puebla contaba con 29,756 unidades económicas en el área de servicios de preparación de alimentos y bebidas, lo que representaba el $5.35 \%$ a nivel nacional; hoy solo se cuenta con el registro de 27,306 unidades, es decir, $8 \%$ menos que el año anterior, por lo que resulta de vital importancia la reactivación inmediata del sector con estrategias oportunas y adecuadas para un ingreso a la nueva normalidad bajo estándares de seguridad y protección, tanto para clientes internos como externos en los diversos establecimientos del ramo. 
Ya desde finales de agosto de 2020, el gobierno del Estado de Puebla autorizó la reapertura de los restaurantes (aquellos que lograron sobrevivir), con ciertas restricciones de servicios, horario y aforo, sin embargo, es de primordial importancia conocer la situación y postura de los consumidores con respecto a su decisión de acudir a los establecimientos, tomando como referencia las medidas sanitarias que aseguren su estancia y experiencia, y haciendo uso de los medios adecuados para llegar y dar a conocer a los comensales la situación de los restaurantes, así pues, se deben proponer estrategias de mercadotecnia que solventen las necesidades del ramo frente a la nueva normalidad.

Mercadotecnia gastronómica para la industria restaurantera

Kotler y Keller (2009) mencionan que la mercadotecnia es un proceso social que consiste en identificar deseos y necesidades, formulando objetivos para satisfacerlos, y estrategias que creen un valor superior para la retención del consumidor y que den fortaleza a las empresas frente a sus competidores, es decir, a través del uso de estrategias de mercadotecnia, adecuadas al contexto de la nueva normalidad, se podrá lograr el reposicionamiento de los restaurantes en Puebla.

Las estrategias de mercadotecnia aplicadas de forma adecuada contribuyen a alcanzar mayores niveles de competitividad que ayudan a las empresas a crear valor y desarrollar ventajas para sobresalir sostenidamente en el competido mercado meta, adaptándose a las contingencias del entorno (Sánchez-Gutiérrez, Vázquez-Ávila, \& Mejía-Trejo, 2017; González, Vizcarra \& Guerrero, 2018) que, y es que en la nueva normalidad es apremiante contar con herramientas que permitan a los restaurantes atraer clientes e incrementar sus ventas, con al intención de recuperar e incluso superar de forma gradual la competitividad con la que contaban previo al inicio de la pandemia por COVID-19.

De acuerdo a Rochat (como se citó en Cabrera, 2013) existen tres elementos para lograr la continuidad en un negocio gastronómico: conocer y manejar los costos, abordar el aprendizaje de un comportamiento profesional y la aparición de un nuevo tipo de consumidor, y es justamente en este último, en el que la creación y uso de estrategias de mercadotecnia, ayudarán a la industria restaurantera de la Ciudad de Puebla, a entender los cambios generados a partir de la pandemia, proponiendo tendencias y métodos que permitan satisfacer las necesidades y deseos de los comensales ante el panorama de la nueva normalidad.

Los esfuerzos para la creación de estrategias de mercadotecnia en el ramo restaurantero en la Ciudad de Puebla, deberán estar enfocados en la preservación de experiencias seguras y placenteras para los comensales, que 
les permitan disfrutar de los alimentos y servicios intangibles que cada establecimiento les pueda ofrecer.

Con base en un estudio realizado en la Ciudad de Puebla con una muestra de 600 participantes pertenecientes a la generación Z (26 años de edad o menos), se definieron las necesidades que requieren ser cubiertas por los restaurantes para atraer y retener clientes, mismas que fueron clasificadas de acuerdo a las estrategias de mercadotecnia establecidas por González, Vizcarra y Guerrero (2018). En la tabla 1 se presentan las necesidades de los comensales pertenecientes a la generación $\mathrm{Z}$ durante la pandemia por Covid19.

Tabla 1. Necesidades de los comensales pertenecientes a la generación Z durante la pandemia por Covid-19

\begin{tabular}{|c|c|c|c|}
\hline Estrategia & Dimensión & Indicador & Necesidades \\
\hline $\begin{array}{l}\text { Penetración } \\
\text { de mercado }\end{array}$ & $\begin{array}{l}\text { Imagen } \\
\text { corporativa }\end{array}$ & $\begin{array}{l}\text { Publicidad } \\
\text { medios }\end{array}$ & 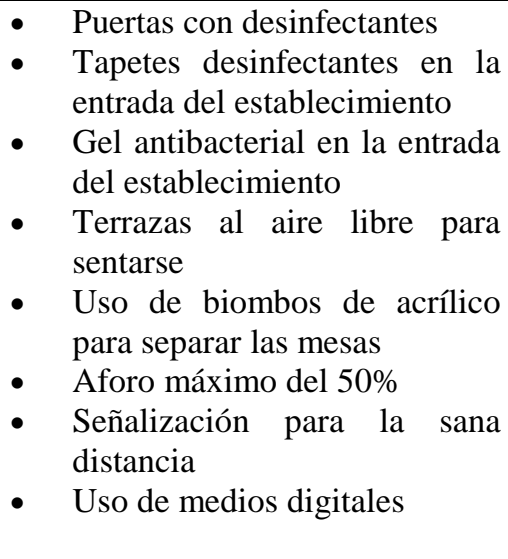 \\
\hline $\begin{array}{l}\text { Desarrollo } \\
\text { de mercado }\end{array}$ & $\begin{array}{l}\text { Atención } \\
\text { clientes }\end{array}$ & $\begin{array}{l}\text { Evaluación } \\
\text { atención }\end{array}$ & $\begin{array}{ll}\text { - } & \text { Uso de guantes por parte del } \\
& \text { personal } \\
\text { - } & \text { Tomar la temperatura al al } \\
\text { ingresar al establecimiento } \\
\text { - } \\
\text { - Pago con tarjeta } \\
\text { Preferencia por no existencia } \\
\text { de valet parking } \\
\text { - Personal de sala con uniforme } \\
\text { - Sana distancia } \\
\text { Sanitización constante de } \\
\text { mobiliario y sanitarios } \\
\end{array}$ \\
\hline $\begin{array}{l}\text { Desarrollo } \\
\text { de productos }\end{array}$ & $\begin{array}{l}\text { Mejoramiento } \\
\text { de productos }\end{array}$ & $\begin{array}{lr}\text { Higiene en } & \text { la } \\
\text { preparación } & \text { de } \\
\text { alimentos } & y \\
\text { bebidas } & \end{array}$ & $\begin{array}{l}\text { - } \begin{array}{l}\text { Alimentos y bebidas } \\
\text { preparados por personal con }\end{array} \\
\text { cubrebocas y lavado de manos } \\
\text { constante } \\
\text { - Traslado de alimentos con una } \\
\text { tapa de acero inoxidable } \\
\text { - } \begin{array}{l}\text { Preferencia por platillo } \\
\text { calientes } \\
\text { - Preferencia por bebidas } \\
\text { embotelladas }\end{array}\end{array}$ \\
\hline
\end{tabular}




\begin{tabular}{llll}
$\begin{array}{l}\text { Presentación del } \\
\text { menú } \\
\text { Presentación de } \\
\text { mesa }\end{array}$ & $\begin{array}{l}\text { Menú digital } \\
\text { Preferencia por ver cómo y con } \\
\text { qué limpian la mesa }\end{array}$ \\
& $\begin{array}{l}\text { Montaje de cubiertos a la } \\
\text { llegada del comensal y en } \\
\text { empaque cerrado }\end{array}$ \\
\hline
\end{tabular}

Elaboración propia con información obtenida de González, Vizcarra y Guerrero (2018).

La generación Z y su consumo durante la pandemia de COVID- 19

La necesidad del hombre por comprender las diferencias que hay entre los distintos grupos poblacionales, lo ha llevado a clasificarla en generaciones. Una generación es un grupo de personas que comparten determinados años de nacimiento así como diferentes eventos significativos que pudieran ser determinantes para su estilo de vida (Kupperschmidt, 2000). En la actualidad, la generación $\mathrm{Z}$ es la que se denomina como la más joven, es decir, son todas aquellas personas que nacieron después de 1995 según Cilliers (2017), teniendo la característica distintiva de ser la generación que ha sido criada durante los años 2000 y que ha experimentado cambios significativos en lo que respecta a la digitalización, la accesibilidad a la información que ofrece la World Wide Web (www) con dispositivos como laptops, teléfonos inteligentes, entre otros.

Además, otra característica fundamental, es que esta generación puede combinar el mundo virtual y el mundo real recalcando su complementariedad (Dolot, 2018).

Junto con la generación X (personas nacidas entre 1980 y 1995) la generación $\mathrm{Z}$ es práctica y comparten fortalezas frente al conocimiento de la tecnología, así como un mayor grado de confort frente al mundo. Sin embargo, la generación Z, se han destacado por ser grandes consumidores.

Entre las tendencias que busca esta generación se destacan características como la innovación, la conveniencia, la seguridad y el escape de la realidad (Wood, 2013).

En el caso de las tendencias gastronómicas, y hasta antes de la pandemia, esta población por influencia de generaciones anteriores, se preocupaba por que los alimentos que consumía fueran considerados como saludables entre los que destacaban los sustitutos de carne, las bebidas con bajo o sin contenido de alcohol e inclusive productos sustentables (Watrous, 2020). Sin embargo, se prevé que, durante la pandemia, las personas en general regresen a tendencia de consumo anteriores ya que estos productos generan una sensación de familiaridad en esta época de incertidumbre.

En el 2018, de acuerdo con McSweeney, los consumidores que pertenecen a la generación $\mathrm{Z}$ son los responsables de hasta un cuarto del tráfico de las visitas a los restaurantes. Esto puede significar que están dispuestos a 
pagar por la experiencia, siempre y cuando, se toman en cuenta factores importantes como atmósfera, mercadotecnia y el menú.

La generación Z o también llamada "zoomers" en Estados Unidos, buscan entre las tendencias de los restaurantes lo siguiente; que dentro del menú se encuentre comida "comida de la calle", las comidas con pollo, los alimentos a base de plantas, los alimentos fermentados, aperitivos o snacks y los "bowls" (pasta, ensalada y aderezos) (McSweeney, 2019).

Según Lara y Ortega (2017), la generación Z o "iGeneration" es una generación que está habituada a las compras, la toma de decisiones y aportación de opiniones "en línea" y estas también pueden ayudar a adquirir o no bienes o servicios.

De acuerdo con los estudios realizados por grandes marcas, el ecommerce se ha visto cada vez más fortalecido. Hay diferentes maneras en las cuales las empresas atienden las necesidades de sus compradores; por un lado, esta el comercio en línea de productos que también pueden adquirirse de manera física y la compra de productos que sólo pueden ser adquiridos de manera digital, siendo ambos una gran alternativa para captar la atención de sus compradores.

Otra tendencia de consumo es la personalización de los servicios en el menor tiempo posible por lo que el uso de los smartphones la clave de las transacciones. De tal forma, el 60\% de los usuarios efectúan compras mediante estos dispositivos, el $78 \%$ consulta precios y el $81 \%$ los utiliza para realizar comparaciones entre productos (Elogia, 2015).

Las experiencias de compra son ahora un objetivo diferenciador y de inversión de las marcas en donde conceptos como transparencia, personalización de productos y servicios y autenticidad son claves en su éxito. En un estudio realizado por la empresa consultora PriceWaterhouseCooper (2012), explica que para que las empresas pueden tener influencia sobre las personas, deben de aprender a escuchar y a publicitar sus productos en las distintas redes sociales de forma distinta para cada una ya que aproximadamente el $75 \%$ de las personas entrevistadas mencionan que sus preferencias de consumo se basan en las opiniones de personas no expertas por lo que el contacto con los denominados "influencers" sería fundamental para el posicionamiento de marca.

Los mensajes "de impacto" serían desde ahora los mensajes más utilizados por las empresas para captar la atención de la GenTech (conocida también como Generación Z), debido a los 8 segundos de atención concreta que pueden tener, a la dificultad para hablar en público y a su incapacidad de escribir textos complejos. Por esto, los rasgos de diferenciación de estos se pueden nombrar como las 5 i's y las 5 c's: Influencia y conexión, inmediatez y cortoplacismo, innovación y creatividad, Irreverencia y contraste y finalmente, inquietud y cambio (Lara \& Ortega, 2017). 
El marketing de influencia es la tendencia entre esta gran área. Se basa en identificar a las personas clave (influencers digitales o bloggers) que tienen influencia sobre un gran número de personas para que comuniquen el mensaje de la marca o de la empresa, y que la combinación de las redes sociales con la publicidad llegan a un nicho de mercado específico. Sin embargo, Castelló (2016) menciona que todos somos "influencers" sobre nuestra red de personas ya que tanto la satisfacción como la insatisfacción de los productos y servicios que obtenemos es comunicada a través de las diferentes redes sociales.

Entre las redes sociales más utilizadas por la Generación Z (quienes representan un aproximado del $25 \%$ de la población) son, según el periódico español ABC (2018), Instragram, WhatsApp y Youtube. Sin embargo, un gran porcentaje de los usuarios emplea FaceBook e Instagram para la búsqueda y posterior compra de productos

Percepción de la Generación Z para un consumo seguro en restaurantes de Puebla

El impacto de la generación $\mathrm{Z}$ en la sociedad es innegable e imparable. Pero ¿qué hay de su impacto en el ámbito restaurantero y el miedo al regreso a consumir alimentos en establecimientos de moda después de haber sufrido una pandemia y adaptarse a los protocolos de seguridad de la nueva normalidad?

Este cambio ha venido a desestabilizar a la industria restaurantera, la cual está resintiendo el impacto económico que posee la generación $\mathrm{Z}$ ya que su poder adquisitivo y de decisión están relativamente limitados.

Casi la totalidad de los $\mathrm{Z}$ aún viven con sus padres, y aunque son capaces de presionar para ir a los restaurantes que les gustan, no siempre lo consiguen cuando se trata de ir en familia o en su caso solos, ya que el temor de la familia los conduce a quedarse en casa.

Cabe mencionar que la generación $\mathrm{Z}$ cuando salen con amigos, se gastan entre 500 y 600 pesos en una comida (Amaro \& Rodríguez, 2016)

Una de sus fuentes de inspiración más importantes a la hora de elegir el lugar a dónde van acudir son las redes sociales, como auténticos nativos digitales. Los $\mathrm{Z}$ eligen los restaurantes que sus amigos o ídolos recomiendan en Facebook, YouTube, Instagram y Twitter. Pero la pregunta es ¿Por qué? Y la respuesta es... porque en estas redes sociales predomina el vídeo, que es su formato preferido debido a que son muy visuales, esto les ha ayudado a emplear el protocolo de sanidad e higiene en su vida cotidiana y aplicarlo en los establecimientos a los que acuden a degustar alimentos.

Es impresionante para los empleadores de los restaurantes cuando van a tomar nota a una mesa de una generación $\mathrm{Z}$ que acaba de entrar al establecimiento, y ver que ya tienen decidido lo que van a pedir sin apenas mirar la carta a través del código QR, suelen decir que ya saben lo que quieren 
porque lo han visto en Instagram, previo a la llegada al lugar acordado (Williams, 2018). Los $\mathrm{Z}$ son una de las generaciones más exigentes que se han conocido hasta ahora porque desde siempre lo han tenido todo, incluso antes de pedirlo (Burkhard \& Fuggle, 2016).

No es un problema su corta edad, aunque no saben mucho de calidad gastronómica pero debido a su acceso ilimitado a las fuentes de información online, esta generación sabe más que las anteriores sobre lo que ellos consideran "the good food", la comida de verdad, esa que consideran que se tendría que comer más (Burkhard \& Fuggle, 2016).

El retorno a las actividades de entretenimiento puede que no sea inmediato, pues con la llegada de la pandemia esto ha detenido a la industria restaurantera, pero en el futuro, los restaurantes que quieran conquistar a los $\mathrm{Z}$ tendrán que claudicar ante estas tres premisas (o al menos alguna de ellas): redes sociales, experiencias paralelas y calidad gastronómica y/o saludable. En definitiva: no resistirse al cambio y a lo que exigen los jóvenes de esta generación (Duarte, 2016; Münch \& Ricalde, 2015).

Debido a que los nativos de internet son emprendedores y autodidactas, dedican su tiempo libre a acciones que les parecen enriquecedoras, relacionadas con su vocación y es debido a ello que han podido mantenerse en pie ante esta situación de emergencia y aunado a el uso de la tecnología los orilló a pedir comida a domicilio, aunque su preocupación es no conocer a personas y relacionarse, además de endeudarse en estos momentos de incertidumbre por lo que su retorno al consumo de alimentos y bebidas ha sido cauteloso. Sin duda les preocupa el futuro, tienen una cierta sensación de inestabilidad por haber nacido en una economía en crisis y marcada por los efectos de un problema de Salud Pública.

Esta preocupación y su sobreexposición a toneladas de información, les conduce a la creencia arraigada de que tenemos que cambiar la forma en la que comemos antes de que sea demasiado tarde.

\section{Metodología}

Se realizó un estudio transversal, no experimental y un cuestionario en línea de 20 ítems basados en el Protocolo para la implementación de medidas preventivas ante COVID-19 en restaurantes de la Secretaría de protección civil y gestión integral de riesgos de la Ciudad de Puebla y Protocolo de prevención COVID-19 "Mesa segura" de la CANIRAC; distribuida durante cuatro semanas, del 1 del septiembre al 1 de octubre de 2020, mediante muestreo no probabilístico por conveniencia a personas de 26 o menos años de edad (Generación z). Se incluyeron 600 de 616 participantes de la Ciudad de Puebla. La encuesta se desarrolló por medio de Google forms y se distribuyó vía redes sociales. 
El cuestionario está integrado por la sección de datos generales y hábitos de consumo en restaurantes con 4 ítems, la primera variable: penetración de mercado, integrada por 2 dimensiones cuenta con 7 ítems; la segunda variable: desarrollo de mercado con 1 dimensión, tiene 4 ítems; y la tercera: desarrollo de productos, con 2 dimensiones se conforma por 5 ítems.

Resultados y discusión

Con base en la participación de 616 personas, se tomaron como referencia las respuestas de 600 pertenecientes a la GenTech (26 años de edad o menores), de los cuales el $53 \%$ fueron del sexo femenino y el $43 \%$ restante del masculino.

Con respecto a las preferencias de consumo en restaurantes el $73.7 \%$ indicaron que antes de la pandemia acudían de 1 a 3 veces por semana, el $14.9 \%$ ninguna vez y el $11.3 \% 4$ veces o más; del mismo modo el $60.7 \%$ indicaron que en sus visitas a los restaurantes acudían con 1 o 2 acompañantes, $39.3 \%$ con 3 o más acompañantes. Esto concuerda con estudio realizado por Revenuemanage.com (2020) destacó que la mayoría de los consumidores pertenecientes a la generación $\mathrm{Z}$ son frecuentes en restaurantes con 5 visitas o más por semana. Se observó también mediante la misma encuesta que la mayoría de los jóvenes cambiaron su consumo a "para llevar" en comparación con la entrega a domicilio. Dado las características optimistas de esta generación, se espera que después de la pandemia incrementen sus salidas a cenar.

De igual forma, el $65.9 \%$ estarían dispuestos a consumir en restaurantes en este año, sin embargo, el $41.2 \%$ prefieren comer solo lo preparado en casa, seguido del 24.7 que gusta de hacer uso del servicio a domicilio y el $19.8 \%$ de pedir comida para llevar. Lo anterior concuerda con lo encontrado en una encuesta realizada en el 2020 en donde se observó que el $41 \%$ de las personas consumirán más alimentos, aún después de que pase la pandemia, mediante medios digitales. Además de que el $48 \%$ de las personas encuestas mencionó que seguirán comprando utilizando esta vía, por motivo de salud. De igual forma, la mayoría de las personas expresaron que compraran en establecimientos cercanos a su domicilio para apoyar la economía local (Monitor Deloitte, 2020).

Estos datos concuerdan con lo encontrado por Monitor Deloitte (2020) ya que se propone que la propuesta hoy en día, en una "nueva realidad" es un mayor consumo de productos y servicios por medios digitales en comparación con las vías más tradicionales.

Con respecto a la disponibilidad de los participantes para acudir a un restaurante, el $42 \%$ menciona que lo hará cuando el semáforo se encuentre en verde, $25.6 \%$ en amarillo y $21.8 \%$ cuando exista una vacuna, en todas las 
circunstancias, siempre y cuando se cuenten con las medidas de higiene necesarias.

De igual forma, con respecto al método de pago, la gran mayoría. el $51.9 \%$, prefiere pago con tarjeta. De igual forma, Se observó que durante la pandemia, la mayoría de las personas que no habían utilizado pagos en línea, comenzaron a utilizar el pago mediante su dispositivo electrónico destacando su conveniencia y motivos de salud en comparación con el ahorro de tiempo. Sin embargo, se refirieron a este método de pago como inseguro o "falto de transparencia" en comparación con el pago mediante tarjeta de bancaria (EZB, 2020).

Adicionalmente, la Generación Z buscará en mayor proporción, y una vez terminada la pandemia, mejores experiencias por parte de los restaurantes así como mejor accesibilidad, dejando de lado la seguridad y la sanitación por parte del establecimiento (situación que dan por sentado) (Revenuemanage, 2020).

Se destaca que, los restaurantes y en general todos los establecimientos que sirven comida deben de redefinir su estrategia de oferta de productos debido a que es prioridad por todos los rangos de edad, mantener la salud y minimizar el riesgo de contagio a toda costa (Rappeport \& Smialek, 2020). Por lo que a continuación se presentan las estrategias propuestas en esta investigación.

Estrategias de mercadotecnia gastronómica para los restaurantes

Como se ha establecido, la generación $\mathrm{Z}$ se distingue por la búsqueda de experiencias únicas que les permitan acumular emociones, por lo que a continuación se presentan las estrategias de mercadotecnia sugeridas para los restaurantes de la Ciudad de Puebla, enfocadas a satisfacer las necesidades de los centenialls frente a la pandemia:

1. Implementar las estrategias de seguridad e higiene propuestas en la tabla 1 del presente estudio.

2. Brindar experiencias que permitan a los comensales acumular momentos y emociones únicos: considerando no solo los alimentos y bebidas, si no el servicio, la ambientación y decoración en un marco de seguridad e higiene.

3. Uso de medios digitales y redes sociales: con la intención de continuar con la mejora de la experiencia de los clientes aún sin que éstos visiten físicamente el restaurante, tomando en cuenta su preferencia por adquirir alimentos y bebidas con servicio a domicilio o para llevar.

4. Optimización de las opciones de pago sin contacto: Habilitación de terminales de pago con tarjetas bancarias, transferencias o pagos virtuales por medio de páginas web. 
5. Creación de lealtad: por medio de promociones, descuentos, acceso a servicios exclusivos a clientes o estrategias que generen la sensación de un buen aprovechamiento del dinero que pagan los clientes.

6. Resaltar la responsabilidad social y ambiental del establecimiento: como elemento imprescindible para satisfacer las necesidades de los clientes pertenecientes a la Generación Z.

Con respecto a las estrategias de mercadotecnia que se proponen, las encuestas establecidas pro el portal evercom (2020) destaca que el marketing online se convierte en tendencia general creciendo considerablemente la cantidad de videos cortos. De igual forma, en un trabajo realizado por Toledo y Armas (2020), se establecen algunos tipos de mercadotécnica que ayudarían a las empresas en esta época de pandemia como mantener canales digitales para que los productos estén disponibles, la contratación de grupal para entregas a domicilio, así como incentivar a los clientes para que creen su propio contenido (prosumer) y la publicación de protocolos de seguridad y bioseguridad.

En relación a la estrategia de generación de nuevas experiencias, el portal de mercadotecnia Accenture (2020), propone la generación de experiencias virtuales así como la generación de experiencias de juego para mantener a los consumidores interesados tanto en el producto como en las experiencias de la marca.

Así mismo, la empresa Gartner (2020) en una de sus publicaciones menciona que ahora más que nunca hay que escuchar a los clientes y mantener la honestidad y claridad entre empresas y consumidores con el propósito de mantener la lealtad de estos y asegurar su permanencia.

En función de lo que indican los comensales con respecto a acudir a los restaurantes durante y después de la pandemia, a continuación, se enlistan sus necesidades en orden de importancia:

1. Personal de sala con uniforme

2. Uso de cubrebocas y caretas por parte del personal (sala y cocina)

3. Limpieza y desinfección constante de sanitarios

4. Entrega de cubiertos en empaques cerrados

5. Aforo máximo del $50 \%$

6. Toma de temperatura al ingresar a los establecimientos

7. Existencia de puertas con desinfectantes

8. Desinfección y montaje de mesas a la vista del comensal

9. Existencia de tapetes desinfectantes a la entrada de los establecimientos

10. Uso de gel o guantes por parte del personal

11. Preferencia por consumo de bebidas embotelladas

12. Inexistencia de valet parking 
13. Terrazas al aire libre para sentarse a consumir

14. Preferencia por el consumo de alimentos calientes

15. Servicio de alimentos con cubierta de acero inoxidable

16. Pago con tarjeta

17. Uso de biombos de acrílico para separar las mesas

18. Menú con código QR

19. Uso de gel antibacterial al ingresar al establecimiento

20. Desinfección del plaqué frente al comensal

21. Desinfección de mesas y sillas cuando salgan los clientes

22. Distintivo $\mathrm{H}$

23. Señalización de sana distancia

Conclusiones

La mercadotecnia gastronómica para brindar experiencias y emociones únicas en restaurantes en la Ciudad de Puebla ante la nueva normalidad, deberá incluir estrategias de Penetración de mercado, tales como Imagen corporativa al interior de los establecimientos y promoción; Desarrollo de mercado como atención a clientes y Desarrollo de productos por medio de mejoramiento de alimentos y bebidas, que permitan a los establecimientos satisfacer las necesidades y expectativas de un segmento tan exigente como la Generación $\mathrm{Z}$.

Este estudio resultó ser importante debido a que se observó que la reactivación de la industria restaurantera post pandemia será paulatina y cautelosa y deberá estar regida por los más altos estándares de seguridad e higiene propuestos por las autoridades sanitarias y solicitados por los centennials.

\section{References:}

1. ABC (8 de junio del 2018). La Generación $Z$ utiliza cada día cuatro redes sociales. Disponible en

https://www.abc.es/tecnologia/redes/abci-generacion-utiliza-cadacuatro-redes-sociales-

201806070136_noticia.html?ref=https:\%2F\%2Fwww.google.com.m $\mathrm{x}$

2. Accenture (2020). COVID-19: 5 human thrust that experiences need to address. How organizations should respond to the never normal. Accedido 18 octubre 2020.

https://www.accenture.com/_acnmedia/Thought-Leadership-

Assets/PDF-2/Accenture-COVID-19-New-Human-Truths-That-

Experiences-Need-To-Address.pdf

3. Amaro L.J. A. \& Rodríguez, R. C.R. (2016). Seguridad en internet.

Paakat: Revista de Tecnología y Sociedad, (11) ,. [Fecha de consulta 
02 de Octubre de 2020]. ISSN:. Disponible en: https://www.redalyc.org/articulo.oa?id=4990/499054323006

4. Aranda, D. (2019). Industria restaurantera, la mayor empleadora del país: CANIRAC. El sol de San Luis.

5. Barton, C., Haywood, J., Jhunjhunwala, P. \& Bhatía, V. (2013). Traveling with Millenials (Informe de investigación de The Boston Consulting Group). Recuperado de https://www.bog.com/domentsfile129974.pdf

6. Bravo- Torres, J. (2011). M-Commerce. Ingenius. Revista de Ciencia y Tecnología, (5),81-91.[fecha de Consulta 06 de Ocubre de 2020]. ISSN: 1390- 650X. Disponible en: https://www.redalyc.org/articulo.oa?id=5055/505554809008

7. Beell, K. (2015). Los diez comportamientos de consumo más reveladores de los posmillenials. Recuperado de http://negocios,pulzoo.com/los-10-comportamientos-de-consumomas-reveladores-de los-posmilenials/

8. Burkhard \& Fuggle (2016). Informe tendencias de turismo 2017, TrekkSoft ebook library. Recuperado de: https://www.trekksoft.com/hubfs/ES_Trends_2017.pdf.

9. Cabrera, S. (2013). Marketing gastronómico. La experiencia de convertir el momento del consumo en un recuerso memorable. Cuaderno 45 Centro de Estudios en Diseño y Comunicación. pp 165174 ISSN 1668-5229

10. Campos, N. (2019). The Millennial Buying Behaviour towards healthy food products in Ireland (Doctoral dissertation, Dublin, National College of Ireland).

11. Carvalho dos Santos Claro, J., Oliviera Fernándes Torres, M., Nascimiento Joao, B.Y. \& Prudencio Tinoco, J. (2015). Carrera, renta y consumo bajo la perspectiva del joven de la generación "Y". Invenio: Revista de Investigación Académica. 18(34). 119-135.

12. Castello, A.M. (2016). El marketing de influencia. Un caso práctico. En Zacipa-Infante, I., Tur-Viñes, V., Segarra-Saavedra, J. Tendencias publicitarias en Iberoamérica. Diálogo de saberes y experiencias. Colección Mundo Digital de Revista Mediterránea de Comunicación. Editorial Universitas, S.A. España. Pp. 49-65.

13. Cilliers, E.J. (2017). The challenge of teaching genera- tion Z. People: International Journal of Social Sciences, 3(1), 188-198. DOI: 10.20319/pijss.2017.31.188198.

14. Chorneukar, M. J. (2014). To Study the Customer Perceptions of Electronic Food Ordering (Magíster en Administración de empresas (MBA). Universidad Pondicherry. 
15. Dolot, A. (2018). The characteristic of Generation Z. E-mentor. 2(74): 44-50.

16. Duarte, F. (2016). Desarrollo de Tiendas Virtuales En Plataformas Especializadas. Recuperado el 07 de Octubre 2020 de http://www.alfadigital.com.ec/blog/desarrollo-de-tiendas-virtualesen- plataformas-especializadas.html.

17. Elogia (2015). VII Estudio Anual de Mobile Marketing. Accedido 30 agosto 2016. http://blog. elogia.net/vii-estudio-anual-de-mobilemarketing-de-iab-spain-y-elogia-2015/

18. España, J., Guevara, D., \& López, H. A. (2020). Estudio descriptivo sobre las estrategias de marketing digital para el Restaurante Macakus durante el periodo de coyuntura Covid-19 en el segundo semestre del 2020 municipio de Pasto Nariño.

19. EZB (2019). Payment Statistics. Accedido 16 de octubre del 2020. http://sdw.ecb.europa.eu/ reports.do?node $=1000004051$

20. Gartner (2020). Adapt your marketing strategies for COVID-19. Accedido 20 de octubre 2020.

https://www.gartner.com/en/marketing/insights/articles/adapt-themarketing-strategy-for-covid-19

21. González, S., Vizcarra, N., \& Guerrero, D. (2018). Las estrategias de mercadotecnia de los food trucks en tijuana: un análisis desde la perspectiva del empresario. Revista de la Facultad de Contaduría y Ciencias Administrativas, Vol. 4 No 7, Pág. 77-85. ISSN: 2448-6051.

22. INEGI (2020). Directorio Estadístico Nacional de Unidades Económicas. https://www.inegi.org.mx/app/mapa/denue/

23. Kotler, P., \& Keller, K. L. (2009). Dirección de marketing. Madrid: Pearson

24. Kupperschmidt, B.R. (2000). Multigeneration Em- ployees: Strategies for Effective Management. Health Care Manager, 19(1), 65-76. DOI: 10.1097/00126450- 200019010-00011.

25. Lozano, L. (20 de julio de 2020). Hasta 1 de cada 4 restaurantes cerraría por COVID-19. Forbes México. www.forbes.com.mx.

26. Molina, P. N., Celi, E., \& Herrera, J. B. F. (2018). Reportaje sobre la nueva tendencia gastronómica que ofrecen los Food truck en el Valle de Los Chillos. Obtenido de Repositorio Digital Universidad de Israel: 157.100.241.244/handle/47000/1591

27. Monitor Deloitte (2020). Impacto of the COVID-19 crisis on shortand medium-term consumer behaviour. Will the COVID-19 crisis have a lasting effect on consumption?. Accedido 14 de octubre del 2020.

https://www2.deloitte.com/content/dam/Deloitte/de/Documents/cons 
umer-business/Impact\%20of\%20the\%20COVID$19 \% 20$ crisis\%20on\%20consumer\%20behavior.pdf

28. Münch \& Ricalde (2015). Nuevos fundamentos de mercadotecnia. Hacia el liderazgo del mercado. México: Trillas.

29. Ponce, J. (2017).Comportamiento de los centennials como consumidores (Artículo en un blog).

30. PWC (2012). enREDados: Cómo hacer rentables las redes sociales, http://www.pwc.es/es/publicaciones/ retail-yconsumo/assets/enredados-como-hacer-rentables-las-redessociales.pdf

31. Rappeport, A. \& Smialek, J. (14 de abril 2020). The New York Times. https://www.nytimes.com/2020/04/14/us/politics/coronaviruseconomy-recession-depression.html

32. Raziel, Z. (28 de julio de 2020). Pandemia por COVID-19 provocó el cierre de 90 mil restaurantes, reporta Canirac. Animal Político. https://www.animalpolitico.com.

33. Revenuemanage.com (2020). COVID-19 Impact on restaurant conusmers. Accedido 13 de octubre del 2020. https://www.revenuemanage.com/wp-content/uploads/2020/07/RMSInsights-COVID19-ImpactRestaurantConsumers-July2020-

GenerationalInsights.pdf

34. Sánchez-Gutiérrez, J., Vázquez-Ávila, G., \& Mejía-Trejo, J. (2017). La mercadotecnia y los elementos que influyen en la competitividad de las mipymes comerciales en Guadalajara, México. Innovar, 27(65), 93-106. doi: 10.15446/innovar.v27n65.65064.

35. Toledo, A. \& Armas, N. (2020). Tips de Marketing en épocas de COVID y POST COVID. CienciAmérica. 9(2), 1-9.

36. Watrous, M. (4 de junio del 2020). How Covid-19 will affect 2020 food trends. Food Business News. Disponible https://www.foodbusinessnews.net/articles/15762-how-covid-19will-affect-2020-food-trends

37. Williams, L. A. (2018). Dirigiendo el consumo de alimentos y bebidas a los Millenials. Industria Alimenticia, 29(4), 31-32.

38. Wood, S. (2013). Generation $\mathrm{Z}$ as consumers: trends and innovation. Institute for Emerging Issues. Disponible en https://iei.ncsu.edu/wpcontent/uploads/2013/01/GenZConsumers.pdf 